\title{
The Extremal Permanental Sum for a Quasi-Tree Graph
}

\author{
Tingzeng Wu $\mathbb{i D}^{1}$ and Huazhong $\mathrm{Lü}^{2}$ \\ ${ }^{1}$ School of Mathematics and Statistics, Qinghai Nationalities University, Xining, Qinghai 810007, China \\ ${ }^{2}$ School of Mathematical Sciences, University of Electronic Science and Technology of China, Chengdu, Sichuan 610054, China
}

Correspondence should be addressed to Tingzeng Wu; mathtzwu@163.com

Received 25 March 2019; Accepted 15 May 2019; Published 20 May 2019

Academic Editor: Dimitri Volchenkov

Copyright (C) 2019 Tingzeng $\mathrm{Wu}$ and Huazhong Lü. This is an open access article distributed under the Creative Commons Attribution License, which permits unrestricted use, distribution, and reproduction in any medium, provided the original work is properly cited.

Let $G$ be a graph and $A(G)$ the adjacency matrix of $G$. The permanent of matrix $(x I-A(G))$ is called the permanental polynomial of $G$. The permanental sum of $G$ is the sum of the absolute values of the coefficients of permanental polynomial of $G$. Computing the permanental sum is \#p-complete. In this note, we prove the maximum value and the minimum value of permanental sum of quasi-tree graphs. And the corresponding extremal graphs are also determined. Furthermore,we also determine the graphs with the minimum permanental sum among quasi-tree graphs of order $n$ and size $m$, where $n-1 \leq m \leq 2 n-3$.

\section{Introduction}

The permanent of $n \times n$ matrix $M=\left(b_{i j}\right)(i, j=1,2, \ldots, n)$ is defined as

$$
\operatorname{per}(M)=\sum_{\sigma} \prod_{i=1}^{n} b_{i \sigma(i)}
$$

where the sum is taken over all permutations $\sigma$ of $\{1,2, \ldots, n\}$.

Let $G$ be a graph with $n$ vertices and let $A(G)$ be its adjacency matrix. The permanental polynomial of $G$ is defined as

$$
\pi(G, x)=\operatorname{per}(x I-A(G))=\sum_{k=0}^{n} b_{k}(G) x^{n-k},
$$

where $I$ is the unit matrix of order $n$. Basic theory of permanental polynomials is well studied recently in [1-3] and the references therein. Kasum et al.[4] and Merris et al. [5] gave the coefficients of the permanental polynomial of $G$, i.e.,

$$
b_{k}(G)=(-1)^{k} \sum_{H} 2^{c(H)}, \quad 0 \leq k \leq n,
$$

where the sum is taken over all Sachs subgraphs $H$ of $G$ on $k$ vertices and $c(H)$ is the number of cycles in $H$. Recall that a
Sachs subgraph is a graph in which each component is a single edge or a cycle.

The permanental sum of graph $G$, denoted by $P S(G)$, can be defined as the summation of all absolute values of coefficients of permanental polynomial of $G$, i.e.,

$$
P S(G)=\sum_{i=0}^{n}\left|b_{i}(G)\right|=\sum_{i=0}^{n} \sum_{H} 2^{c(H)} .
$$

Thus, $P S(G)=1$ if $G$ is an empty graph. Wu and So [6] have shown that computing permanental sum of a graph is \#Pcomplete.

The permanental sum of a graph was first considered by Tong [7]. In [8], Xie et al. captured a labile fullerene $C_{50}\left(D_{5 h}\right)$. Tong computed all 271 fullerenes in $C_{50}$. In his study, Tong found that the permanental sum of $C_{50}\left(D_{5 h}\right)$ achieves the minimum among all 271 fullerenes in $C_{50}$. He pointed that the permanental sum would be closely related to stability of molecular graphs. Recently, the permanental sum of a graph has received much attention. Li et al. [9] determined the extremal hexagonal chains with respect to permanental sum. $\mathrm{Li}$ and Wei [10] proved the lower and upper bounds for the permanental sum of an octagonal chain. Wu and Lai [11] systematically introduced the properties of permanental sum of a graph. 
A connected graph $G$ is called a quasi-tree graph, if there exists a vertex $u^{*}$ in $G$ such that $G-u^{*}$ is a tree. Let $G$ be a quasi-tree graph with $n$ vertices and $m$ edges. Then $n-1 \leq$ $m \leq 2 n-3$, and the degree of $u^{*}$ in $G$ equals $m-n+2$. Denote $\mathscr{G}_{n}=\{G: G$ is a quasi-tree graph of order $n\}$, and $\mathscr{G}_{n, m}=$ $\{G: G$ is a quasi-tree graph of order $n$ and size $m\}$. As an important class of graphs, quasi-tree graphs have been widely studied. For the background and some known results about quasi-tree graphs, we refer the reader to [12-15].

The purpose of this note is to investigate the properties of permanental sum of quasi-tree graphs. The note is organized as follows. In the next section, we review some previous results that will be needed in the sequel. In Section 3, we discuss the permanental sum of quasi-tree graphs.

\section{Some Preliminary}

In this note, we only consider finite, undirected, and simple graph. Let $G$ be a graph with vertex set $V(G)$ and edge set $E(G)$. The neighborhood of vertex $v \in V(G)$, denoted by $N_{G}(v)$, is the set of vertices adjacent to $v$. The graph that arises from $G$ by deleting a vertex $u \in V(G)$ or an edge $u v \in E(G)$ will be denoted by $G-\mathcal{u}$ or $G-u v$. Let $G+H$ denote the union of two vertex disjoint graphs $G$ and $H$. For any positive integer $l, l G$ denotes the union of $l$ disjoint copies of $G$. The path, cycle, and star of order $n$ are denoted by $P_{n}, C_{n}$ and $K_{1, n-1}$, respectively.

Two edges of $G$ are said to be independent if they are not adjacent in $G$. A $k$-matching of $G$ is a set of $k$ mutually independent edges. For an integer $k \geq 0$, let $m(G, k)$ denote the number of $k$-matchings of a graph $G$. The Hosoya index $Z(G)$ of a graph $G$ is defined to be the total number of matchings of $G$, that is,

$$
Z(G)=\sum_{k=0}^{\lfloor n / 2\rfloor} m(G, k),
$$

where $n$ is the number of the vertices of the graph $G$. Some results on Hosoya indices were studied in [3, 16-18].

For $n \geq 2$, let $F(n)=F(n-1)+F(n-2)$ denote the sequence of Fibonacci numbers, in particular, $F(0)=0$ and $F(1)=1$.

Lemma 1 (see [10]). Let $G=T_{1} \cup T_{2} \cup \ldots \cup T_{t}$ be a forest with order $n \geq 2$ and $t \geq 2$, where $T_{i}$ is a tree with $n_{i}$ vertices, $i=1,2, \ldots, t$. Then $Z(G) \leq \prod_{i=1}^{t} F\left(n_{i}+1\right)$ with equality if and only if $T_{i} \cong P_{n_{i}}$. Moreover $Z(G) \leq F\left(m_{1}+1\right) F\left(m_{2}+1\right)$, where $m_{1}+m_{2}=n$ with equality if and only if $G \cong P_{m_{1}} \cup P_{m_{2}}$.

Let $E_{k}$ be the empty graph of order $k$. Denote $\vee$ the graph joint of two graphs, and $\cup$ the disjoint union of two graphs. The graphs $F_{n}^{m}=\left(K_{1, m-n+1} \cup E_{2 n-m+3}\right) \vee E_{1}$ and $H_{n}^{n+2}=\left(C_{3} \cup\right.$ $\left.E_{n-4}\right) \vee E_{1}$ are shown in Figure 1[19].

Lemma 2 (see [19]). Let $\mathscr{B}_{n, m}$ be the set consisting of all graphs of order $n$ and size $m$. For $G \in \mathscr{B}_{n, m}$ with $n-1 \leq m \leq 2 n-3$,

$$
Z\left(F_{n}^{m}\right) \leq Z(G) .
$$

Equality holds if and only if $G=F_{n}^{m}$, or $H_{n}^{n+2}$ when $m=n+2$, where Graphs $F_{n}^{m}$ and $H_{n}^{n+2}$ see Figure 1.
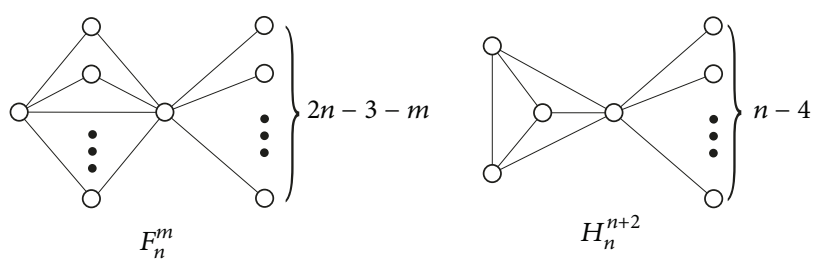

FIGURE 1: Graphs $F_{n}^{m}$ and $H_{n}^{n+2}$ in Lemma 2.

By the definitions of $\mathscr{B}_{n, m}$ and $\mathscr{G}_{n, m}$, we obtain that $\mathscr{G}_{n, m} \subset \mathscr{B}_{n, m}$. By Lemma 2, we have the following.

Corollary 3. Let $G \in \mathscr{G}_{n, m}$ be a quasi-tree graph with $n-1 \leq$ $m \leq 2 n-3$. Then

$$
Z\left(F_{n}^{m}\right) \leq Z(G),
$$

where the equality holds if and only if $G=F_{n}^{m}$.

Lemma 4 (see [11]). Letting $T$ be a tree with order $n \geq 1$, then $n \leq P S(T) \leq F(n+1)$, the first equality holds if and only if $T \cong K_{1, n-1}$, and the second equality holds if and only if $T \cong P_{n}$.

Lemma 5 (see [11]). Let $P_{n}$ be a path with $n$ vertices. Then

$$
P S\left(P_{n}\right)= \begin{cases}1 & \text { if } n=0, \\ 1 & \text { if } n=1, \\ F(n+1) & \text { if } n \geq 2 .\end{cases}
$$

Lemma 6 (see [11]). The permanental sum of a graph satisfies the following identities:

(i) Let $G$ and $H$ be two connected graphs. Then

$$
P S(G \cup H)=P S(G) P S(H) .
$$

(ii) Let $e=u v$ be an edge of a graph $G$ and $\mathscr{C}(e)$ the set of cycles containing $e$. Then

$$
\begin{aligned}
P S(G)= & P S(G-e)+P S(G-v-u) \\
& +2 \sum_{C_{k} \in \mathscr{C}(e)} P S\left(G-V\left(C_{k}\right)\right) .
\end{aligned}
$$

(iii) Let $v$ be a vertex of a graph $G$ and $\mathscr{C}(v)$ the set of cycles containing $v$. Then

$$
\begin{aligned}
P S(G)= & P S(G-v)+\sum_{u \in N_{G}(v)} P S(G-v-u) \\
& +2 \sum_{C_{k} \in \mathscr{C}(v)} P S\left(G-V\left(C_{k}\right)\right) .
\end{aligned}
$$

By Lemma 6, we have the following.

Corollary 7. Let $G$ be a graph and e an edge of $G$. Then PS(Ge) $<P S(G)$. 


\section{Main Results}

In this section, we will investigate the properties of permanental sum of a quasi-tree graph.

Theorem 8. Let $G \in \mathscr{G}_{n, m}$ be a quasi-tree graph with $n-1 \leq$ $m \leq 2 n-3$. Then

$$
m^{2}-m n+m+n \leq P S(G),
$$

where the equality holds if and only if $G \cong F_{n}^{m}$.

Proof. By the definition of permanental sum of a graph, it can be known that $P S(G)=Z(G)+2 w(G)$, where $w(G)$ denotes the number of all Sachs graphs containing cycles of $G$. Checking $G$, we know that $G$ has exactly $\left(\begin{array}{c}d_{G}\left(u^{*}\right) \\ 2\end{array}\right)$ cycles and $d_{G}\left(u^{*}\right)=m-n+2$. Thus $\left(\begin{array}{c}d_{G}\left(u^{*}\right) \\ 2\end{array}\right)=w\left(F_{n}^{m}\right) \leq w(G)$. By Corollary 3 , we have $P S\left(F_{n}^{m}\right) \leq P S(G)$ with equality if and only if $G \cong F_{n}^{m}$. By Lemma 6 , we obtain that

$$
\begin{aligned}
P S\left(F_{n}^{m}\right)= & P S\left(K_{1, m-n+1}\right) \\
& +(2 n-3-m) P S\left(K_{1, m-n+1}\right) \\
& +(m-n+1) P S\left(K_{1, m-n}\right)+1 \\
& +2\left(\begin{array}{c}
m-n+2 \\
2
\end{array}\right)=m^{2}-m n+m+n .
\end{aligned}
$$

This completes the proof.

Theorem 9. Let $G \in \mathscr{G}_{n}$. Then

$$
\begin{aligned}
P S(G) \leq & F(n)+\sum_{i=1}^{n-1} F(i) F(n-i) \\
& +2 \sum_{r=1}^{n-2} \sum_{j_{r}=1}^{n-r-1} F\left(j_{r}\right) F\left(n-r-j_{r}\right),
\end{aligned}
$$

where the equality holds if and only if $G \cong G^{*}$.

Proof. Let $G \in \mathscr{G}_{n}$, and let $u \in N_{G}\left(u^{*}\right)$ and $\mathscr{C}_{G}\left(u^{*}\right)=\{C$ : $C$ is a cycle containing $u^{*}$ in $\left.G \in \mathscr{G}_{n}\right\}$. Suppose that $G \in \mathscr{G}_{n}$ has the maximum permanental sum. We will characterize the structure of $G$. By (iii) of Lemma 6, it can be known that if $G \in \mathscr{G}_{n}$ has the maximum permanental sum, then $P S\left(G-u^{*}\right)$, $\sum_{u^{*} \in N_{G}(v)} P S\left(G-u^{*}-u\right)$, and $\sum_{C_{k} \in \mathscr{C}\left(u^{*}\right)} P S\left(G-V\left(C_{k}\right)\right)$ must attain maximum value. From the definition of a quasi-tree graph, we know that $G-u^{*}$ is a tree. By Lemma $4, G-u^{*}$ has the maximum permanental sum when $G-u^{*}$ is isomorphic to path $P_{n-1}$. Since a path has exactly two vertices of degree 1 . Thus there exist exactly two vertices, say $u^{\prime}$ and $u^{\prime \prime}$, such that $G-u^{*}-u^{\prime}$ and $G-u^{*}-u^{\prime \prime}$ are paths. Set $u \in N_{G}\left(u^{*}\right)-\left\{u^{\prime}, u^{\prime \prime}\right\}$. By Lemma 1 , only when $G-u^{*}-u$ has two components, each of which is a path, does $\sum_{u \in N_{G}\left(u^{*}\right)-\left\{u^{\prime}, u^{\prime \prime}\right\}} P S\left(G-u^{*}-u\right)$ attain the maximum value. Similarly, by Lemma 1 and Lemma 6 , $\sum_{C_{k} \in \mathscr{C}\left(u^{*}\right)} P S\left(G-V\left(C_{k}\right)\right)$ attains the maximum value if and only if $G-V\left(C_{k}\right)$ has two components, each of which is a path, and $G$ has the largest number of cycles in $\mathscr{G}_{n}$. Combining

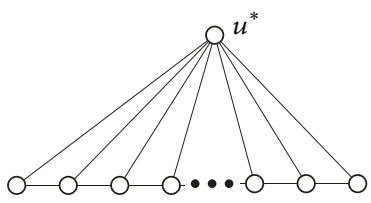

$G^{*}$

Figure 2: Graph $G^{*}$.

arguments above and Corollary 7, $G$ must be isomorphic to $G^{*}$ (see Figure 2). Let the number of 3-cycles, 4 -cycles,..., $n$-cycles in $G^{*}$ be $j_{1}, j_{2}, \ldots, j_{r}, \ldots, j_{n-3}, j_{n-2}$, respectively. By Lemma 6, we obtain that

$$
\begin{aligned}
P S & \left(G^{*}\right)=P S\left(P_{n-1}\right)+\sum_{i=1}^{n-1} P S\left(P_{i-1}\right) P S\left(P_{n-1-i}\right) \\
& +2\left(\sum_{j_{1}=1}^{n-2} P S\left(P_{j_{1}-1}\right) P S\left(P_{n-2-j_{1}}\right)\right. \\
& +\sum_{j_{2}=1}^{n-3} P S\left(P_{j_{2}-1}\right) P S\left(P_{n-3-j_{2}}\right) \\
& +\sum_{j_{3}=1}^{n-4} P S\left(P_{j_{3}-1}\right) P S\left(P_{n-4-j_{3}}\right)+\ldots \\
& +\sum_{j_{n-3}=1}^{2} P S\left(P_{j_{n-3}-1}\right) P S\left(P_{n-(n-2)-j_{n-3}}\right) \\
& \left.+P S\left(P_{j_{n-2}-1}\right) P S\left(P_{n-(n-1)-j_{n-2}}\right)\right)=P S\left(P_{n-1}\right) \\
& +\sum_{i=1}^{n-1} F(i) F(n-i)+2 \sum_{r=1}^{n-2} \sum_{j_{r}=1}^{n-r-1} F\left(j_{r}\right) F\left(n-r-j_{r}\right) \\
& +\sum_{i=1}^{n-1} P S\left(P_{i-1}\right) P S\left(P_{n-1-i}\right) \\
& 2 \sum_{j_{r}=1}^{n-2} P S\left(P_{j_{r}-1}\right) P S\left(P_{n-r-1-j_{r}}\right)=F(n)
\end{aligned}
$$

By Theorems 8 and 9, we obtain the following result.

Theorem 10. Let $G \in \mathscr{G}_{n}$. Then

$$
\begin{aligned}
n \leq & P S(G) \\
\leq & F(n)+\sum_{i=1}^{n-1} F(i) F(n-i) \\
& +2 \sum_{r=1}^{n-2} \sum_{j_{r}=1}^{n-r-1} F\left(j_{r}\right) F\left(n-r-j_{r}\right),
\end{aligned}
$$


The first equality holds if and only if $G \cong K_{1, n-1}$, and the second equality holds if and only if $G \cong G^{*}$.

\section{Data Availability}

No data were used to support this study.

\section{Conflicts of Interest}

The authors declare that there are no conflicts of interest regarding the publication of this paper.

\section{Acknowledgments}

This research is supported by the National Natural Science Foundation of China (Nos. 11761056, 11801061); the Ministry of Education Chunhui Project (No. Z2017047) and the NSF of Qinghai Province (No. 2016-ZJ-947Q); and the Key Project of QHMU (No. 2019XJZ10).

\section{References}

[1] Y. Shi, M. Dehmer, X. Li, and I. Gutman, Graph Polynomials, CRC Press, 2016.

[2] T. Wu and H.-J. Lai, "On the permanental nullity and matching number of graphs," Linear and Multilinear Algebra, vol. 66, no. 3, pp. 516-524, 2018.

[3] G. Yu and H. Qu, "The coefficients of the immanantal polynomial," Applied Mathematics and Computation, vol. 339, pp. 3844, 2018.

[4] D. Kasum, N. Trinajstić, and I. Gutman, "Chemical graph theory. III. On permanental polynomial," Croatica Chemica Acta, vol. 54, pp. 321-328, 1981.

[5] R. Merris, K. R. Rebman, and W. Watkins, "Permanental polynomials of graphs," Linear Algebra and its Applications, vol. 38, pp. 273-288, 1981.

[6] T. Wu and W. So, "Unicyclic graphs with second largest and second smallest permanental sums," Applied Mathematics and Computation, vol. 351, pp. 168-175, 2019.

[7] H. Tong, Parallel Algorithms for Computing Permanents and Permanental Polynomials of Sparse Matrices [PhD. thesis], Tsinghua University, 2006.

[8] S.-Y. Xie, F. Gao, X. Lu et al., "Capturing the labile fullerene[50] as $\mathrm{C}_{50} \mathrm{Cl}_{10}$," Science, vol. 304, no. 5671, p. 699, 2004.

[9] W. Li, Z. Qin, and H. Zhang, "Extremal hexagonal chains with respect to the coefficients sum of the permanental polynomial," Applied Mathematics and Computation, vol. 291, pp. 30-38, 2016.

[10] S. Li and W. Wei, "Extremal octagonal chains with respect to the coefficients sum of the permanental polynomial," Applied Mathematics and Computation, vol. 328, pp. 45-57, 2018.

[11] T. Wu and H.-J. Lai, "On the permanental sum of graphs," Applied Mathematics and Computation, vol. 331, pp. 334-340, 2018.

[12] S. Li, X. Li, and W. Jing, "On the extremal Merrifield-Simmons index and Hosoya index of quasi-tree graphs," Discrete Applied Mathematics, vol. 157, no. 13, pp. 2877-2885, 2009.

[13] M. Lu and J. Gao, "On the Randić index of quasi-tree graphs," Journal of Mathematical Chemistry, vol. 42, no. 3, pp. 297-310, 2007.
[14] X.-F. Pan, X. Liu, and H. Liu, "On the spectral moment of quasitrees," Linear Algebra and its Applications, vol. 436, no. 5, pp. 927-934, 2012.

[15] K. Xu, Z. Zheng, and K. C. Das, "Extremal $t$-apex trees with respect to matching energy," Complexity, vol. 21, pp. 238-247, 2016.

[16] L. Chen, J. Liu, and Y. Shi, "Matching energy of unicyclic and bicyclic graphs with a given diameter," Complexity, vol. 21, no. 2, pp. 224-238, 2015.

[17] W. Liu, Q. Guo, Y. Zhang, L. Feng, and I. Gutman, "Further results on the largest matching root of unicyclic graphs," Discrete Applied Mathematics, vol. 221, pp. 82-88, 2017.

[18] S. Wagner and I. Gutman, "Maxima and minima of the Hosoya index and the Merrifield-Simmons index: a survey of results and techniques," Acta Applicandae Mathematicae, vol. 112, no. 3, pp. 323-346, 2010.

[19] W. So and W.-H. Wang, "Finding the least element of the ordering of graphs with respect to their matching numbers," MATCH - Communications in Mathematical and in Computer Chemistry, vol. 73, no. 1, pp. 225-238, 2015. 


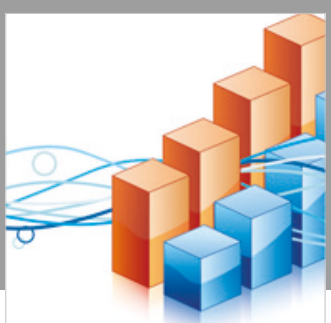

Advances in

Operations Research

\section{-n-m}
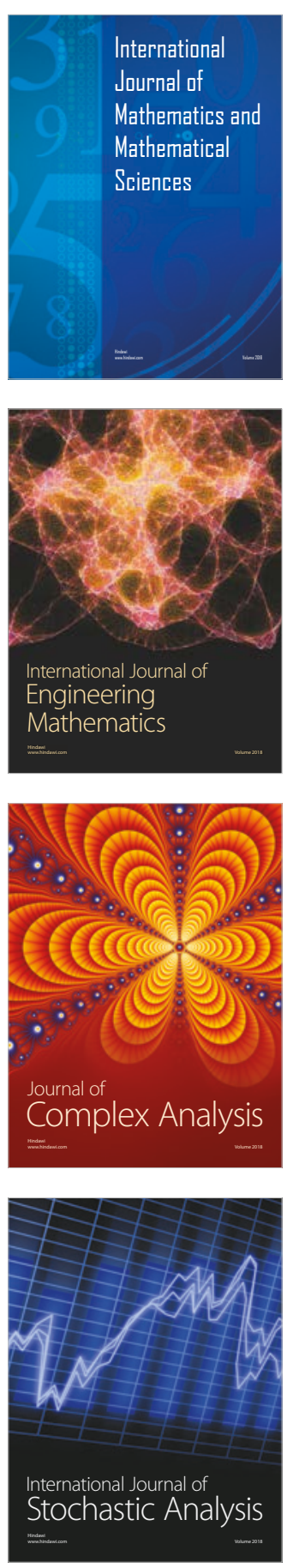
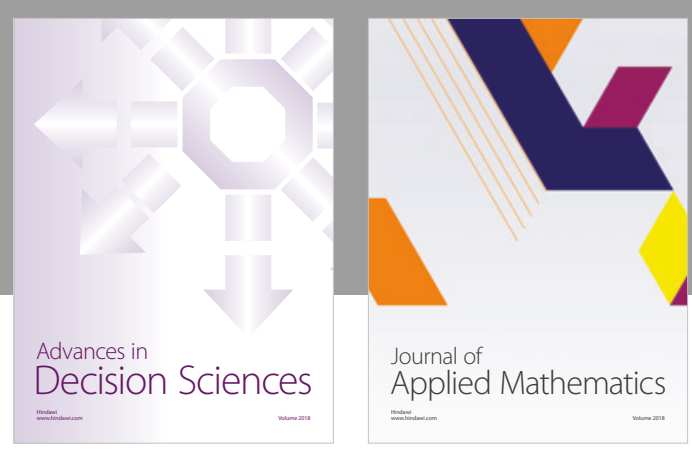

Journal of

Applied Mathematics
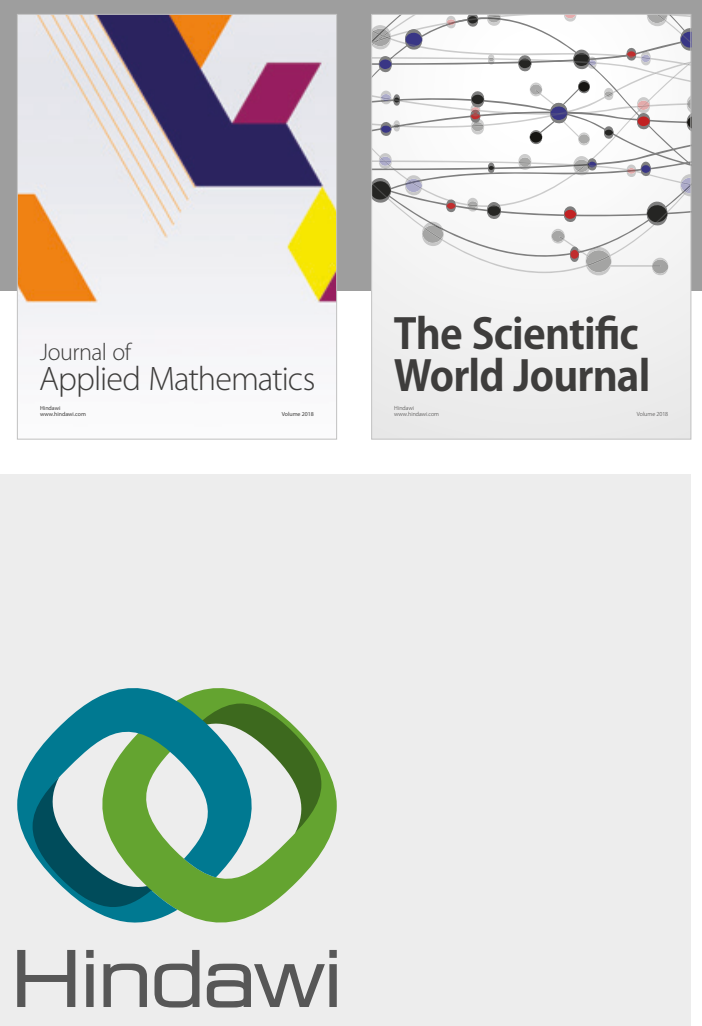

Submit your manuscripts at

www.hindawi.com

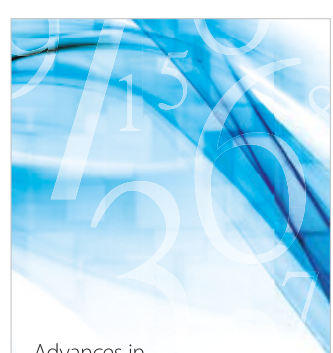

Advances in
Numerical Analysis
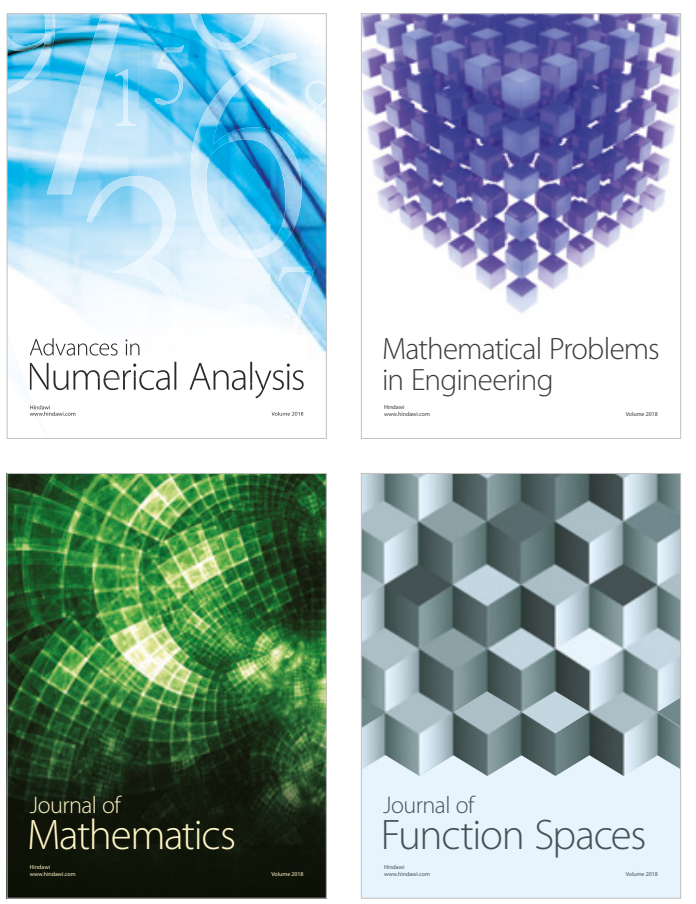

Mathematical Problems in Engineering

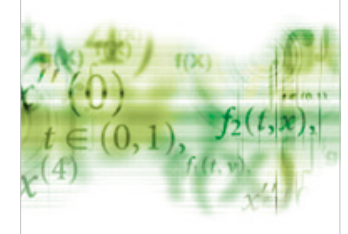

International Journal of

Differential Equations

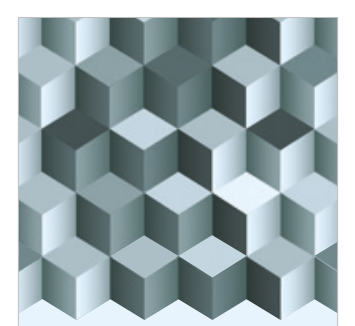

Journal of

Function Spaces
The Scientific

World Journal

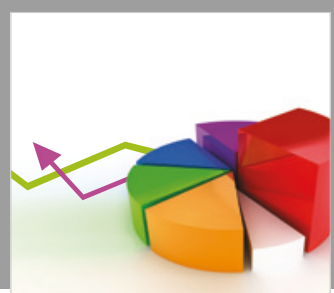

Journal of

Probability and Statistics
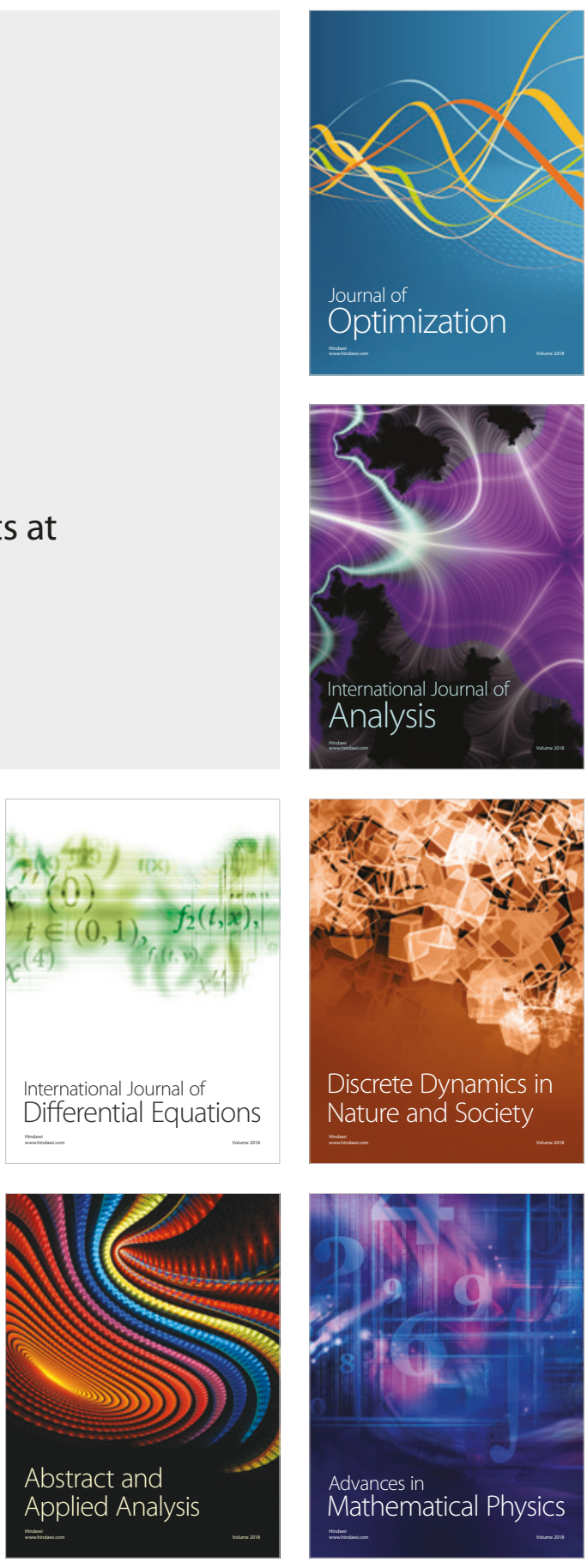